\title{
Iatrogenic aortic dissection caused by incorrect frozen elephant trunk deployment
}

\author{
Yaojun Dun ${ }^{1}$ and Xiaogang Sun ${ }^{2}$ \\ ${ }^{1}$ Affiliation not available \\ ${ }^{2}$ Fuwai Hospital State Key Laboratory of Cardiovascular Disease
}

October 8, 2020

\begin{abstract}
We reported a rare case of iatrogenic aortic dissection caused by incorrect frozen elephant trunk (FET) deployment. A 53-yearold man underwent total arch replacement with FET for aortic arch aneurysm and concurrent PAU in the descending aorta. However, after FET deployment, aortic dissection occurred. We analyzed that the tip of FET stent had been inserted into the PAU and caused aortic dissection. We performed some salvage procedures. However, the patient died on the 1st postoperative day. Therefore, care should be taken to avoid incorrect deployment of FET when there is a large tear or ulcer lesion in the proximal descending aorta.
\end{abstract}

\section{Introduction}

The reports regarding the management of the aortic arch aneurysm with concurrent penetrating atherosclerotic ulcer (PAU) in the descending aorta are very limited. It could be managed by different approaches. In this report, we treated a case with total arch replacement (TAR) with frozen elephant trunk (FET). However, iatrogenic aortic dissection occurred after FET deployment into the PAU. And we analyzed the possible causes and summarized some points to prevent this complication. The study was approved by the institutional review board of Fuwai Hospital. A waiver of informed consent was granted due to the design of the study.

\section{Case Report}

A 53-year-old man came to our center. He complained of chest pain and shortness of breath for 9 days. Computed tomography (CT) scan showed an aortic arch aneurysm with a PAU in the descending aorta, right pleural effusion and right lower lobe atelectasis (Figure 1A, 1B). The PAU was detected in the descending aorta at about $100 \mathrm{~mm}$ from the left subclavian artery on CT scan. We had taken active medical treatment for him, including anti-infection, red blood cell transfusion, analgesia, anti-hypertension. 10 days later, coronary CT scan showed that the number of PAU increased and diameter of PAU elevated (Figure 1C). And he underwent open surgery one day later.

Under hypothermic circulatory arrest, we performed TAR with a four-branched vascular graft $(28 \times 90 \mathrm{~mm}$; Terumo, Vascutek Limited, Renfrewshire, UK) after a FET stent $(28 \times 120 \mathrm{~mm}$; Cronus, MicroPort Endovascular Shanghai Co, Ltd, China) was deployed into the descending aorta under direct vision. After TAR with FET, the arterial pressure of the left radial artery was higher than that of the left dorsalis pedis, with a difference of about $40 \mathrm{mmHg}$. We performed aortography via the right femoral artery. However, it was very difficult for the guidewire to move forward. And aortography only showed the narrow thoracic aorta resembled a thin line (Figure 2A). Then we had to perform antegrade aortography via one branch stump of the vascular graft. Only thoracoabdominal aorta, bilateral intercostal arteries and lumbar arteries could be found from the scan, while the celiac axis, superior mesenteric artery, and renal arteries disappeared (Figure 
2B). We comprehensively analyzed that the tip of the FET had been inserted into the PAU and caused iatrogenic aortic dissection. The true lumen of thoracoabdominal aorta was totally obliterated and viscera were ischemic. We performed ascending aorta-right femoral artery bypass at once, but the true lumen of aorta did not dilate. Then we performed endovascular thoracoabdominal aortic fenestration to connect the true lumen and the false lumen (Figure 2C). And a stent graft $(28 \times 80 \mathrm{~mm}$; Valiant, Medtronic Vascular, Santa Rosa, CA, USA) was retrogradely deployed to the descending aorta to restore the perfusion of the true lumen (Figure 2D). After that, the radial artery and the dorsalis pedis pressures were equal. But these procedures were complicated, and it took about four hours to complete these procedures. During this period, the visceral including liver, intestine and kidneys were ischemic. Progressive lactic acidosis developed during operation. The patient received bedside hemofiltration intraoperatively. After he was returned to the intensive care unit, the hemodynamics deteriorated quickly. And the patient died of multiorgan failure on the $1^{\text {st }}$ postoperative day.

\section{Discussion}

For the aortic arch aneurysm with concurrent PAU in the descending aorta, there are many approaches, including TAR with FET, type I hybrid arch repair (arch debranching in native aorta zone 0 and TEVAR) and type II hybrid arch repair (arch debranching in Dacron zone 0 after ascending aorta replacement and TEVAR) (1), TEVAR with chimney technique, and hemiarch and descending aorta replacement via thoracotomy $(2)$. In this case, the diameter of ascending aorta was $41 \mathrm{~mm}$. Considering the ascending aorta more than $40 \mathrm{~mm}$ is associated with a high risk of retrograde ascending aortic dissection after TEVAR(3), we abandoned type I hybrid arch repair and TEVAR with chimney technique. And if we performed type II hybrid arch repair, at least two TEVAR stent grafts were needed, and the medical expenses were expensive for the patient. Moreover, hemiarch and descending aorta replacement via thoracotomy shows worse outcomes when comparing with TAR(2). Therefore, we performed type III arch repair for this patient.

In this case, a FET stent with a length of $120 \mathrm{~mm}$ was selected to cover the PAU, because the distance from the PAU to the left subclavian artery was about $100 \mathrm{~mm}$. But there may exist little deviation when measuring the distance on CT scan. And the tip of the FET stent is very sharp. Therefore, we analyzed that the tip of the FET stent was possibly at the same level with the PAU after deployment, and the tip may have been inserted into the PAU and caused iatrogenic aortic dissection. The Microport FET system was routinely used for TAR at our center. The delivery system enables direct insertion without a guidewire. And the FET stent had been correctly deployed in all cases. It was a rare case with incorrect FET deployment.

From this case, we could conclude that attention should be taken to avoid incorrect deployment of FET. We had also summarized some points to prevent this complication, especially in patients with a large tear or ulcer lesion in the proximal descending aorta. Firstly, the FET stent could be deployed with a guidewire under direct vision. The guidewire could also be retrogradely placed through femoral artery. Secondly, early detection of iatrogenic aortic dissection was very important. The radial artery pressure and the femoral artery or the dorsalis pedis pressure should be closely monitored intraoperatively to identify aortic dissection. And serum lactic acid should also be closely monitored to detect visceral malperfusion. Thirdly, the distance from the left subclavian artery to the primary tear or ulcer lesion in the descending aorta should be measured carefully on CT scan. If the distance was very close to the length of the commercially available FET stent $(100 \mathrm{~mm}, 120 \mathrm{~mm})$, we could adopt type II hybrid arch repair. Because retrograde deployment of TEVAR stent through a guidewire under X-ray is very safe. Moreover, distal arch and descending aorta replacement via thoracotomy could also be adopted in this situation.

Once iatrogenic aortic dissection had happened, some salvage procedures should be performed. If the aortic dissection was detected early, we could remove the suture lines and take out the FET stent under hypothermic circulatory arrest, then re-deploy the FET stent into the true lumen of the descending aorta. Besides, endovascular aortic fenestration and additional stent deployment would be the suitable option. But the fenestration technique was very difficult and carried high risks(4). And open fenestration could also be adopted when the other strategies were not available(5). 


\section{Conflict of interest: none declared.}

\section{References:}

1. Bavaria J, Vallabhajosyula P, Moeller P, Szeto W, Desai N, Pochettino A. Hybrid approaches in the treatment of aortic arch aneurysms: postoperative and midterm outcomes. J Thorac Cardiovasc Surg. 2013;145(3 Suppl):S85-90.

2. Joo HC, Youn YN, Lee SH, Lee S, Chang BC, Yoo KJ. Clinical outcomes of different surgical approaches for proximal descending thoracic aneurysm involving the distal arch. J Thorac Cardiovasc Surg. 2018;155(6):2289-98 e1.

3. Williams JB, Andersen ND, Bhattacharya SD, Scheer E, Piccini JP, McCann RL, et al. Retrograde ascending aortic dissection as an early complication of thoracic endovascular aortic repair. J Vasc Surg. 2012;55(5):1255-62.

4. Norton EL, Williams DM, Kim KM, Khaja MS, Wu X, Patel HJ, et al. Management of acute type B aortic dissection with malperfusion via endovascular fenestration/stenting. J Thorac Cardiovasc Surg. 2019.

5. Trimarchi S, Segreti S, Grassi V, Lomazzi C, Cova M, Piffaretti G, et al. Open fenestration for complicated acute aortic B dissection. Ann Cardiothorac Surg. 2014;3(4):418-22.

Figure 1: (A)(B) CT scan after admission; (C) CT scan 10 days later. Arrows represented the PAU of the descending aorta, and asterisk represented the aortic arch aneurysm.

Figure 2: (A) Aortography showed that the narrow thoracic aorta resembling a thin line. (B) Aortography showed the thoracoabdominal aorta, bilateral intercostal arteries and lumbar arteries, while the celiac axis, superior mesenteric artery and renal arteries disappeared. (C) Endovascular thoracoabdominal aortic fenestration was performed to connect the true lumen and the false lumen. (D) Stent graft was retrogradely deployed to the descending aorta.

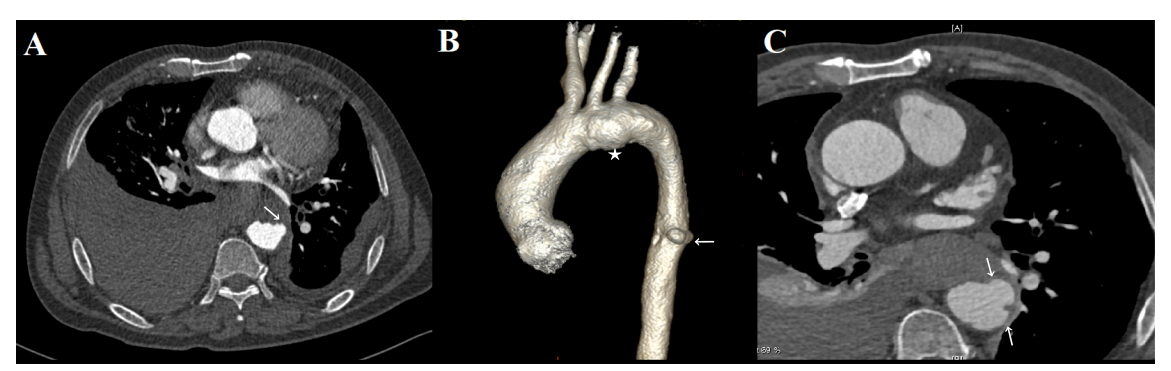




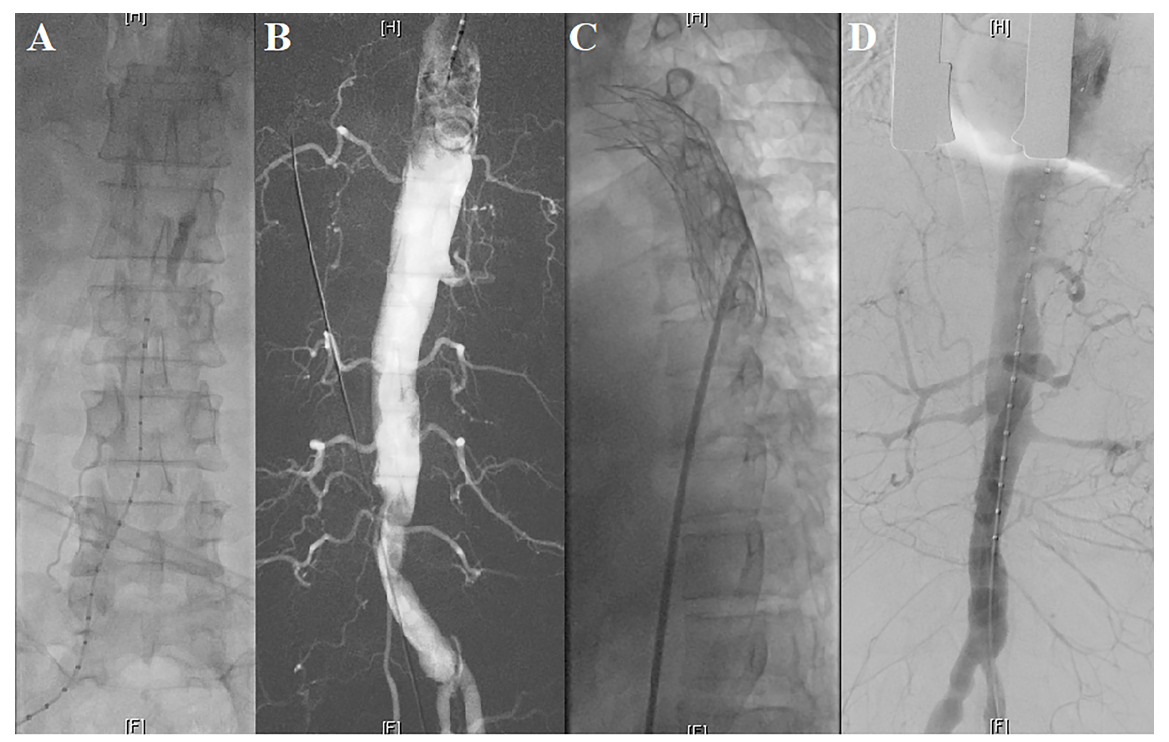

